\title{
Grid-based methods for diatomic quantum scattering problems III: Double photoionization of molecular hydrogen in prolate spheroidal coordinates
}

\author{
Liang Tao*, ${ }^{1}$ C. W. McCurdy, ${ }^{1,2}$ and T. N. Rescigno ${ }^{1}$ \\ ${ }^{1}$ Lawrence Berkeley National Laboratory, Chemical Sciences, Berkeley, CA 94720 \\ ${ }^{2}$ Departments of Applied Science and Chemistry, University of California, Davis, CA 95616
}

(Dated: September 28, 2011)

\begin{abstract}
Our previously developed finite-element/ discrete variable representation in prolate spheroidal coordinates is extended to two-electron systems with a study of double ionization of $\mathrm{H}_{2}$ with fixednuclei. Particular attention is paid to the development of fast and accurate methods for treating the electron-electron interaction. The use of exterior complex scaling in the implementation offers a simple way of enforcing Coulomb boundary conditions for the electronic double continuum. While the angular distributions calculated in this study are found to be completely consistent with our earlier treatments that employed single-center expansions in spherical coordinates, we find that the magnitude of the integrated cross sections are sensitive to small changes in the initial-state wave function. The present formulation offers significant advantages with respect to convergence and efficiency and opens the way to calculations on more complicated diatomic targets.
\end{abstract}

PACS numbers: 33.80.E, 31.15.A

\section{INTRODUCTION}

The implementation of advanced nonperturbative methods for solving either the time-independent [1-3] or time-dependent [4-6] Schrödinger equation has made it possible to obtain essentially exact wave functions on a numerical grid for atoms with two active electrons. These methods have been particularly successful for studying the response of simple atoms when exposed to intense, ultrashort xuv laser pulses $[7,8]$. While there have been some efforts to extend such studies to simple diatomic molecules in recent years [9-11], the nonspherical nature of the interaction potentials necessarily complicates the implementation. Indeed, fully differential double ionization cross sections on molecule other than $\mathrm{H}_{2}$ have yet to appear.

In molecular applications, some consideration should be given to the underlying representation that is used: for diatomic targets, prolate spheroidal coordinates are a natural choice, as we have shown in two earlier papers $[12,13]$ (referred to hereafter as I and II, respectively) where we developed a finite-element/ discrete variable representation (FEM/DVR) in those coordinates and presented results for the single-electron case of $\mathrm{H}_{2}^{+}$. Our purpose here is to extend the FEM/DVR treatment in prolate spheroidal coordinates to the case of molecular hydrogen. As in our earlier studies of $\mathrm{H}_{2}$ double ionization based on single-center expansions in spherical coordinates $[9,14,15]$ we use exterior complex scaling (ECS)[1] to simplify the imposition of outgoingwave Coulomb boundary conditions. In this connection, we must mention the recent work of Serov and Joulakian [16], who have also studied $\mathrm{H}_{2}$ double ion-

\footnotetext{
*Present address: Department of Chemistry, University of Southern California, Los Angeles, CA 90089
}

ization in prolate spheroidal coordinates using an ECS method. Their prolate spheroidal exterior complex scaling (PSECS) approach differs from ours by using a Bspline, rather than FEM/DVR, basis to expand the wave function, as well as a different prescription for computing the one-electron $\left(\mathrm{H}_{2}^{+}\right)$testing functions that are needed to construct the double ionization amplitudes. The numerical results we obtain are somewhat different from theirs, as are the conclusions about convergence of the methods.

We will show that the use of FEM/DVR gives simple formulas for constructing matrix elements of the electronic repulsion potential, $1 /\left|\mathbf{r}_{1}-\mathbf{r}_{2}\right|$ which are diagonal in the indices corresponding to the FEM/DVR mesh points. These properties, combined with the more rapid convergence of the wave function in prolate spheroidal coordinates than in spherical coordinates, give a very efficient method for treating the electronic double continuum with diatomic targets.

The outline of this paper is as follows. The theoretical treatment is outlined in Sec. II. Section III details the basis set and grid parameters used in the numerical calculations and Sec. IV presents results and comparisons with earlier work. We conclude with a brief discussion.

\section{THEORY}

\section{A. FEM/DVR for $\mathbf{H}_{2}$ in prolate spheroidal coordinates}

Procedures for constructing a grid-based set of functions using finite-elements and the DVR in prolate spheroidal coordinates, along with explicit formulas for matrix elements of all required one-body operators, have been given in I, so only a brief summary will be given here. The electronic Hamiltonian for $\mathrm{H}_{2}$ with fixed inter- 
nuclear vector $\mathbf{R}$ is given as (atomic units throughout)

$$
\begin{aligned}
H\left(\mathbf{r}_{1}, \mathbf{r}_{2}\right)= & -\frac{1}{2} \nabla_{1}^{2}-\frac{1}{2} \nabla_{2}^{2}-\frac{1}{\left|\mathbf{r}_{1}-\frac{\mathbf{R}}{2}\right|}-\frac{1}{\left|\mathbf{r}_{1}+\frac{\mathbf{R}}{2}\right|} \\
& -\frac{1}{\left|\mathbf{r}_{2}-\frac{\mathbf{R}}{2}\right|}-\frac{1}{\left|\mathbf{r}_{2}+\frac{\mathbf{R}}{2}\right|}+\frac{1}{\left|\mathbf{r}_{1}-\mathbf{r}_{2}\right|}
\end{aligned}
$$

Prolate spheroidal coordinates $(\xi, \eta, \phi)$ are defined in the usual way by rotating a two-dimensional elliptical coordinate system $(\xi, \eta)$ about the focal axis of the ellipse:

$$
\begin{array}{ll}
\xi=\frac{\left|\mathbf{r}-\frac{\mathbf{R}}{2}\right|+\left|\mathbf{r}+\frac{\mathbf{R}}{2}\right|}{R} & (1 \leq \xi \leq \infty) \\
\eta=\frac{\left|\mathbf{r}-\frac{\mathbf{R}}{2}\right|-\left|\mathbf{r}+\frac{\mathbf{R}}{2}\right|}{R} & (-1 \leq \eta \leq 1),
\end{array}
$$

where $\phi(0 \leq \phi \leq 2 \pi)$ is the azimuthal angle. The Laplacian in these coordinates is

$$
\begin{gathered}
\nabla^{2}=\frac{4}{R^{2}\left(\xi^{2}-\eta^{2}\right)}\left[\frac{\partial}{\partial \xi}\left(\xi^{2}-1\right) \frac{\partial}{\partial \xi}+\frac{\partial}{\partial \eta}\left(1-\eta^{2}\right) \frac{\partial}{\partial \eta}\right. \\
\left.+\left(\frac{1}{\left(\xi^{2}-1\right)}+\frac{1}{\left(1-\eta^{2}\right)}\right) \frac{\partial^{2}}{\partial \phi^{2}}\right]
\end{gathered}
$$

and the volume element is

$$
d V=(R / 2)^{6}\left(\xi_{1}^{2}-\eta_{1}^{2}\right)\left(\xi_{2}^{2}-\eta_{2}^{2}\right) d \xi_{1} d \xi_{2} d \eta_{1} d \eta_{2} d \phi_{1} d \phi_{2}
$$

while the electron-nuclear attraction is

$$
-\frac{1}{\left|\mathbf{r}-\frac{\mathbf{R}}{2}\right|}-\frac{1}{\left|\mathbf{r}+\frac{\mathbf{R}}{2}\right|}=-\frac{4 \xi}{R\left(\xi^{2}-\eta^{2}\right)} .
$$

The amplitude for double ionization is obtained by solving a driven equation for the first-order wave function obtained when the radiation field is treated perturbatively:

$$
\left(E_{0}+\omega-H\right) \Psi_{s c}^{+}=\boldsymbol{\epsilon} \cdot \boldsymbol{\mu} \Psi_{0},
$$

where $\Psi_{0}$ is the wave function for the initial state of $\mathrm{H}_{2}$ with energy $E_{0}, \omega$ is the photon energy, $\epsilon$ is the polarization unit vector and $\boldsymbol{\mu}$ is the electronic dipole operator. To solve this equation, we expand $\Psi_{s c}^{+}$(and $\Psi_{0}$ ) in a product basis of functions of the electronic coordinates of each electron. For the "angular " variables, we use ordinary spherical harmonics $Y_{l}^{m}(\eta, \phi)$ in the variables $\cos ^{-1}(\eta)$ and $\phi$, while the "radial variables" $\xi_{1}$ and $\xi_{2}$ are discretized using FEM/DVR:

$$
\Psi_{s c}^{+}=\sum_{i, j, l_{1}, l_{2}, m} C_{i j l_{1} l_{2} m} \Phi_{i l_{1} m}\left(\mathbf{r}_{1}\right) \Phi_{j l_{2} M-m}\left(\mathbf{r}_{2}\right)
$$

where

$$
\Phi_{i l m}(\mathbf{r}) \equiv \chi_{i m}(\xi) Y_{l}^{m}(\eta, \phi)
$$

where $\chi_{i m}$ is a DVR basis function and where we have used the fact that the component of total electronic angular momentum, $M$, along the internuclear axis is a good quantum number.
As outlined in I, specification of the elementary DVR functions [17] depends on whether $m$ is even or odd. For even $m$, we chose

$$
\chi_{i m}(\xi)=\frac{1}{\sqrt{w_{i}}} \prod_{j \neq i}^{N} \frac{\xi-\xi_{j}}{\xi_{i}-\xi_{j}}
$$

where $\left(\xi_{j}, w_{j}\right)$ are the points and weights associated with an $N$-point Gauss quadrature. In the FEM/DVR scheme, the $\xi$ grid, which runs from 1 to some specified maximum value $\xi_{\max }$, is divided into subintervals. In the first interval, we use Gauss-Radau quadrature, which excludes the point $\xi=1$ as a quadrature point; Gauss-Lobatto quadrature, which includes the endpoints as quadrature points, is used in the other intervals, which insures continuity across element boundaries.

For odd $m$ the DVR functions are modified to account for the non-analytic behavior of the wave function at $\xi=$ $1[18,19]$ :

$$
\chi_{i m}(\xi)=\frac{\left(\xi^{2}-1\right)^{1 / 2}}{\sqrt{w_{i}\left(\xi_{i}^{2}-1\right)}} \prod_{j \neq i}^{N} \frac{\xi-\xi_{j}}{\xi_{i}-\xi_{j}} .
$$

The DVR functions so defined have the property of discrete orthonormality at the quadrature points,

$$
\chi_{i m}\left(\xi_{j}\right)=\delta_{i j} / \sqrt{w_{i}},
$$

and provide a diagonal representation of any local operator when the matrix elements are evaluated using the underlying Gauss quadrature rule,

$$
\begin{aligned}
\int \chi_{i m}(\xi) f(\xi) \chi_{j m}(\xi) d \xi & \approx \sum_{k} \chi_{i m}\left(\xi_{k}\right) f\left(\xi_{k}\right) \chi_{j m}\left(\xi_{k}\right) w_{k} \\
& =\delta_{i j} f\left(\xi_{i}\right) .
\end{aligned}
$$

However, as noted in I and II, the product basis defined in Eq. (31) is not orthogonal in $l_{1}$ and $l_{2}$ because of the volume element:

$$
\begin{aligned}
<\Phi_{i l_{1} m_{1}} & \Phi_{j l_{2} m_{2}} \mid \Phi_{i^{\prime} l_{1}^{\prime} m_{1}^{\prime}} \Phi_{j^{\prime} l_{2}^{\prime} m_{2}^{\prime}}>= \\
& (R / 2)^{6} \delta_{i i^{\prime}} \delta_{j j^{\prime}} \delta_{m_{1} m_{1}^{\prime}} \delta_{m_{2} m_{2}^{\prime}} S_{m_{1} l_{1} l_{1}^{\prime}}\left(\xi_{i}\right) S_{m_{2} l_{2} l_{2}^{\prime}}\left(\xi_{j}\right)
\end{aligned}
$$

where

$$
\begin{aligned}
& S_{m l l^{\prime}}(\xi) \equiv \int Y_{l}^{m *}(\eta, \phi) Y_{l^{\prime}}^{m}(\eta, \phi)\left(\xi^{2}-\eta^{2}\right) d \eta d \phi \\
& =\delta_{l l^{\prime}}\left(\xi^{2}-\frac{2 l^{2}-2 m^{2}+2 l-1}{(2 l+3)(2 l-1)}\right)-\delta_{l l^{\prime}-2} \frac{1}{(2 l+3)} \\
& \quad \times\left(\frac{(l+m+1)(l-m+1)(l+m+2)(l-m+2)}{(2 l+1)(2 l+5)}\right)^{1 / 2} \\
& -\delta_{l l^{\prime}+2}(2 l+1) \\
& \quad \times\left(\frac{(\ell+m-1)(l-m-1)(l+m)(l-m)}{(2 l-1)(2 l+3)}\right)^{1 / 2}
\end{aligned}
$$

For notational simplicity, we will not display integration limits, with the understanding that the implied limits on $\xi, \eta$ and $\phi$ are $\left[1, \xi_{\max }\right],[-1,1]$ and $[0,2 \pi]$, respectively. 


\section{B. Electron-electron repulsion integrals}

Our strategy for evaluating matrix elements of the electron repulsion operator parallels, as closely as possible, the procedure we developed for FEM/DVR in a spherical basis. The two-electron integral we seek to evaluate is

$$
\begin{aligned}
I \equiv & \int \bar{\Phi}_{i l_{1} m_{1}}\left(\xi_{1} \eta_{1} \phi_{1}\right) \bar{\Phi}_{j l_{2} m_{2}}\left(\xi_{2} \eta_{2} \phi_{2}\right) \frac{1}{\left|\mathbf{r}_{1}-\mathbf{r}_{2}\right|} \\
& \times \Phi_{i^{\prime} l_{1}^{\prime} m_{1}^{\prime}}\left(\xi_{1} \eta_{1} \phi_{1}\right) \Phi_{j^{\prime} l_{2}^{\prime} m_{2}^{\prime}}\left(\xi_{2} \eta_{2} \phi_{2}\right) d V
\end{aligned}
$$

where the bar on $\Phi$ indicates complex conjugation only of its associated spherical harmonic. We begin with the
Neumann expansion of $1 / r_{12}$ in prolate spheroidal coordinates [20]:

$$
\begin{aligned}
\frac{1}{\left|\mathbf{r}_{1}-\mathbf{r}_{2}\right|} & =\frac{8 \pi}{R} \sum_{l=0}^{\infty} \sum_{m=-l}^{l}(-1)^{m} \frac{(l-m) !}{(l+m) !} \\
& \times P_{l}^{m}\left(\xi_{<}\right) Q_{l}^{m}\left(\xi_{>}\right) Y_{l}^{m}\left(\eta_{1}, \phi_{1}\right) Y_{l}^{m *}\left(\eta_{2}, \phi_{2}\right)
\end{aligned}
$$

where $P_{l}^{m}$ and $Q_{l}^{m}$ are the regular and irregular associated Legendre functions [21], respectively. Substituting the Neumann expansion of Eq. (17) into Eq. (16) gives

$$
\begin{aligned}
I=\left(\frac{R}{2}\right)^{6} \frac{8 \pi}{R} \sum_{l=|m|}^{\infty}(-1)^{m} \frac{(l-m) !}{(l+m) !} \int & \chi_{i m_{1}}\left(\xi_{1}\right) \chi_{i^{\prime} m_{1}^{\prime}}\left(\xi_{1}\right) \chi_{j m_{2}}\left(\xi_{2}\right) \chi_{j^{\prime} m_{2}^{\prime}}\left(\xi_{2}\right) \\
& \times P_{l}^{m}\left(\xi_{<}\right) Q_{l}^{m}\left(\xi_{>}\right) J_{l_{1} m_{1} l_{1}^{\prime} m_{1}^{\prime}}^{l m}\left(\xi_{1}\right) \bar{J}_{l_{2} m_{2} l_{2}^{\prime} m_{2}^{\prime}}\left(\xi_{2}\right) d \xi_{1} d \xi_{2}
\end{aligned}
$$

where

$$
\begin{aligned}
& J_{l_{1} m_{1} l_{1}^{\prime} m_{1}^{\prime}}^{l m}(\xi)=\int Y_{l_{1}}^{m_{1} *}(\eta, \phi) Y_{l}^{m}(\eta, \phi) Y_{l_{1}^{\prime}}^{m_{1}^{\prime}}(\eta, \phi)\left(\xi^{2}-\eta^{2}\right) d \eta d \phi \\
& \bar{J}_{l_{2} m_{2} l_{2}^{\prime} m_{2}^{\prime}}^{l m}(\xi)=\int Y_{l_{2}}^{m_{2} *}(\eta, \phi) Y_{l}^{m *}(\eta, \phi) Y_{l_{2}^{\prime}}^{m_{2}^{\prime}}(\eta, \phi)\left(\xi^{2}-\eta^{2}\right) d \eta d \phi
\end{aligned}
$$

Note that there is no sum over $m$ in Eq. (18) since it is fixed by the selection rule $m=m_{1}-m_{1}^{\prime}=m_{2}^{\prime}-m_{2}$.

The angular integrals $J_{l_{1} m_{1} l_{1}^{\prime} m_{1}^{\prime}}^{l m}$ and $\bar{J}_{l_{2} m_{2}}^{l m} l_{2}^{\prime} m_{2}^{\prime}(\xi)$ can be done analytically. Because of the factor $\left(\xi^{2}-\eta^{2}\right)$ in the volume element, the required angular integrals are of two general types:

$$
\begin{aligned}
A_{l_{1} l l_{1}^{\prime}}^{m_{1} m m_{1}^{\prime}}=\int Y_{l_{1}}^{m_{1} *}(\eta, \phi) Y_{l}^{m}(\eta, \phi) Y_{l_{1}^{\prime}}^{m_{1}^{\prime}}(\eta, \phi) d \eta d \phi \\
=(-1)^{m_{1}} \sqrt{\frac{\left(2 l_{1}+1\right)(2 l+1)\left(2 l_{1}^{\prime}+1\right)}{4 \pi}} \\
\quad \times\left(\begin{array}{ccc}
l_{1} & l & l_{1}^{\prime} \\
0 & 0 & 0
\end{array}\right)\left(\begin{array}{ccc}
l_{1} & l & l_{1}^{\prime} \\
-m_{1} & m & m_{1}^{\prime}
\end{array}\right)
\end{aligned}
$$

and

$$
B_{l_{1} l l_{1}^{\prime}}^{m_{1} m m_{1}^{\prime}}=\int \eta^{2} Y_{l_{1}}^{m_{1} *}(\eta, \phi) Y_{l}^{m}(\eta, \phi) Y_{l_{1}^{\prime}}^{m_{1}^{\prime}}(\eta, \phi) d \eta d \phi .
$$

To evaluate the $B$ integrals, we can use $\eta^{2}=\frac{1}{3}+\frac{4}{3} \sqrt{\frac{\pi}{5}} Y_{2}^{0}$ along with the identity

$$
\begin{aligned}
Y_{l}^{m} Y_{2}^{0}= & (-1)^{m} \sum_{j=|l-2|}^{|l+2|} \sqrt{\frac{5(2 l+1)(2 j+1)}{4 \pi}} \\
& \times\left(\begin{array}{lll}
2 & l & j \\
0 & 0 & 0
\end{array}\right)\left(\begin{array}{ccc}
2 & l & j \\
0 & m & -m
\end{array}\right) Y_{j}^{m}
\end{aligned}
$$

to write

$$
\begin{gathered}
B_{l_{1} l l_{1}^{\prime}}^{m_{1} m m_{1}^{\prime}}=\frac{1}{3} A_{l_{1} l l_{1}^{\prime}}^{m_{1} m m_{1}^{\prime}}+\frac{4}{3} \sqrt{\frac{\pi}{5}}(-1)^{m} \\
\sum_{j=|l-2|}^{|l+2|} \sqrt{\frac{5(2 l+1)(2 j+1)}{4 \pi}}\left(\begin{array}{ccc}
2 & l & j \\
0 & 0 & 0
\end{array}\right)\left(\begin{array}{ccc}
2 & l & j \\
0 & m & -m
\end{array}\right) \\
\times A_{l_{1} j l_{1}^{\prime}}^{m_{1} m m_{1}^{\prime}} .
\end{gathered}
$$

The complete angular integrals are

$$
\begin{aligned}
& J_{l_{1} m_{1} l_{1}^{\prime} m_{1}^{\prime}}^{l m}(\xi)=\xi^{2} A_{l_{1} l l_{1}^{\prime}}^{m_{1} m m_{1}^{\prime}}-B_{l_{1} l l_{1}^{\prime}}^{m_{1} m m_{1}^{\prime}} \\
& \bar{J}_{l_{2} m_{2} l_{2}^{\prime} m_{2}^{\prime}}^{l m}(\xi)=(-1)^{m}\left(\xi^{2} A_{l_{2} l l_{2}^{\prime}}^{m_{2}-m m_{2}^{\prime}}-B_{l_{2} l l_{2}^{\prime}}^{m_{2}-m m_{2}^{\prime}}\right)
\end{aligned}
$$

The integrals over the radial variables in Eq. (18) cannot be approximated accurately by Gauss quadrature over the DVR mesh points because of the derivative discontinuity in $P_{l}^{m}\left(\xi_{<}\right) Q_{l}^{m}\left(\xi_{>}\right)$. We can however restore the validity of the underlying quadrature with an approach similar to that employed in our earlier studies that use a spherical coordinate basis [1]. The approach is based on solving Poisson's equation. We begin by defining the radial densities $\rho_{i m_{1} i^{\prime} m_{1}^{\prime}}(\xi) \equiv \chi_{i m_{1}}(\xi) \chi_{i^{\prime} m_{1}^{\prime}}(\xi)$ and $\rho_{j m_{2} j^{\prime} m_{2}^{\prime}}(\xi) \equiv \chi_{j m_{2}}(\xi) \chi_{j^{\prime} m_{2}^{\prime}}(\xi)$. We then define the potential $V_{j j^{\prime}}^{l_{2} m_{2} l_{2}^{\prime} m_{2}^{\prime}}$ at $\mathbf{r}_{1}$ due to the distribution 
$\rho_{j m_{2} j^{\prime} m_{2}^{\prime}} Y_{l_{2}}^{m_{2}} Y_{l_{2}^{\prime}}^{m_{2}^{\prime}}$

$$
V_{j j^{\prime}}^{l_{2} m_{2} l_{2}^{\prime} m_{2}^{\prime}}\left(\mathbf{r}_{1}\right)=\int \frac{\rho_{j m_{2} j^{\prime} m_{2}^{\prime}}\left(\xi_{2}\right) Y_{l_{2}}^{m_{2} *}\left(\eta_{2}, \phi_{2}\right) Y_{l_{2}^{\prime}}^{m_{2}^{\prime}}\left(\eta_{2}, \phi_{2}\right)}{\left|\mathbf{r}_{1}-\mathbf{r}_{2}\right|}\left(\xi_{2}^{2}-\eta_{2}^{2}\right) d \xi_{2} d \eta_{2} d \phi_{2},
$$

so that

$$
\begin{aligned}
I=\int \rho_{i m_{1} i^{\prime} m_{1}^{\prime}} & (\xi) Y_{l_{1}}^{m_{1} *}(\eta, \phi) Y_{l_{1}^{\prime}}^{m_{1}^{\prime}}(\eta, \phi) \\
& \times V_{j j^{\prime}}^{l_{2} m_{2} l_{2}^{\prime} m_{2}^{\prime}}(\xi, \eta, \phi)\left(\xi^{2}-\eta^{2}\right) d \xi d \eta d \phi .
\end{aligned}
$$

Since the Green's function for Poisson's equation in prolate spheroidal coordinates satisfies

$$
\nabla^{2} \frac{1}{\left|\mathbf{r}_{1}-\mathbf{r}_{2}\right|}=-\left(\frac{2}{R}\right)^{3} \frac{4 \pi}{\xi^{2}-\eta^{2}} \delta\left(\xi-\xi^{\prime}\right) \delta\left(\eta-\eta^{\prime}\right) \delta\left(\phi-\phi^{\prime}\right),
$$

it follows that the potential $V_{j j^{\prime}}^{l_{2} m_{2} l_{2}^{\prime} m_{2}^{\prime}}$ satisfies

$$
\begin{aligned}
& \nabla^{2} V_{j j^{\prime}}^{l_{2} m_{2} l_{2}^{\prime} m_{2}^{\prime}}(\mathbf{r})= \\
& \quad-\left(\frac{2}{R}\right)^{3} 4 \pi \rho_{j m_{2} j^{\prime} m_{2}^{\prime}}(\xi) Y_{l_{2}}^{m_{2 *}}(\eta, \phi) Y_{l_{2}^{\prime}}^{m_{2}^{\prime}}(\eta, \phi) .
\end{aligned}
$$

We next expand $V_{j j^{\prime}}^{l_{2} m_{2} l_{2}^{\prime} m_{2}^{\prime}}$ in spherical harmonics:

$$
V_{j j^{\prime}}^{l_{2} m_{2} l_{2}^{\prime} m_{2}^{\prime}}(\mathbf{r})=\sum_{l=0}^{\infty} \sum_{m=-l}^{l} v_{j j^{\prime} ; l m}^{l_{2} m_{2} l_{2}^{\prime} m_{2}^{\prime}}(\xi) Y_{l}^{m}(\eta, \phi) .
$$

Substituting this expansion into Eq. (28), integrating from the left with $\int Y_{l}^{m *}(\eta, \phi)\left(\xi^{2}-\eta^{2}\right) d \eta d \phi$ and using Eq. (3) gives

$$
\begin{gathered}
{\left[\frac{\partial}{\partial \xi}\left(\xi^{2}-1\right) \frac{\partial}{\partial \xi}-l(l+1)-\frac{m^{2}}{\left(\xi^{2}-1\right)}\right] v_{j j^{\prime} ; l m}^{l_{2} m_{2} l_{2}^{\prime} m_{2}^{\prime}}(\xi)=} \\
-\frac{8 \pi}{R} \rho_{j m_{2} j^{\prime} m_{2}^{\prime}}(\xi) \bar{J}_{l_{2} m_{2} l_{2}^{\prime} m_{2}^{\prime}}(\xi)
\end{gathered}
$$

To solve Eq. (30), we begin by expanding $v_{j j^{\prime} ; l m}^{l_{2} m_{2} l_{2}^{\prime} m_{2}^{\prime}}(\xi)$ in the FEM/DVR basis

$$
v_{j j^{\prime} ; l m}^{0 l_{2} m_{2} l_{2}^{\prime} m_{2}^{\prime}}(\xi)=\sum_{i} d_{i} \chi_{i m}(\xi) .
$$

Substituting the above expansion into Eq. (30), multiplying from the left with another DVR basis function and integrating over $\xi$, using the Gauss quadrature rule, gives a set of linear equations that can be solved for the coefficients $[d]$ :

$$
d_{i}=-\frac{8 \pi}{R} \sum_{j}\left[T_{l m}\right]_{i, j}^{-1} \frac{\delta_{j j^{\prime}}}{\sqrt{w_{j}}} \bar{J}_{l_{2} m_{2} l_{2}^{\prime} m_{2}^{\prime}}\left(\xi_{j}\right),
$$

where $\mathbf{T}_{l m}$ is the matrix representation of the operator that appears on the LHS of Eq. (30) in the DVR basis, whose elements are given by:

$$
\begin{aligned}
{\left[T_{l m}\right]_{i, j}=-\int } & \frac{\partial \chi_{i}(\xi)}{\partial \xi} \frac{\partial \chi_{j}(\xi)}{\partial \xi}\left(\xi^{2}-1\right) d \xi \\
& -\delta_{i j}\left(l(l+1)-\frac{m^{2}}{\xi_{i}^{2}-1}\right)
\end{aligned}
$$

The function $v_{j j^{\prime}: l m}^{0 l_{2} m_{2} l_{2}^{\prime} m_{2}^{\prime}}(\xi)$ vanishes at the endpoints of the $\xi$-grid. The physical solution we seek, on the other hand, is finite at the grid endpoint, $\xi_{\max }$. Its value at $\xi_{\text {max }}$, obtained by substituting Eqs. (17) and (31) into Eq. (25), is

$$
\begin{aligned}
v_{j j^{\prime} ; l m}^{0 l_{2} m_{2} l_{2}^{\prime} m_{2}^{\prime}}\left(\xi_{\text {max }}\right)=\frac{8 \pi}{R} Q_{l}^{m}\left(\xi_{\text {max }}\right) \frac{(l-m) !}{(l+m) !} \\
\quad \times \int P_{l}^{m}(\xi) \rho_{j m_{2} j^{\prime} m_{2}^{\prime}}(\xi) \bar{J}_{l_{2} m_{2} l_{2}^{\prime} m_{2}^{\prime}}(\xi) d \xi
\end{aligned}
$$

We can construct a solution with the proper boundary conditions by adding to $v_{j j^{\prime} ; l m}^{0 l_{2} m_{2} l_{2}^{\prime} m_{2}^{\prime}}(\xi)$ components of the homogeneous equation corresponding to Eq. (30), which is just the Legendre equation. We can therefore write the general solution to Eq. (30) as

$$
\begin{aligned}
& v_{j j^{\prime} ; l m}^{l_{2} m_{2} l_{2}^{\prime} m_{2}^{\prime}}(\xi)=v_{j j^{\prime} ; l m}^{0 l_{2} m_{2} l_{2}^{\prime} m_{2}^{\prime}}(\xi) \\
& \quad+\alpha_{j l_{2} m_{2} j^{\prime} l_{2}^{\prime} m_{2}^{\prime}} P_{l}^{m}(\xi)+\beta_{j l_{2} m_{2} j^{\prime} l_{2}^{\prime} m_{2}^{\prime}}^{l m} Q_{l}^{m}(\xi) .
\end{aligned}
$$

We can take $\beta_{j l_{2} m_{2} j^{\prime} l_{2}^{\prime} m_{2}^{\prime}}^{l m}=0$ because the physical solution must be regular at $\xi=1 . \alpha_{j l_{2} m_{2} j^{\prime} l_{2}^{\prime} m_{2}^{\prime}}^{l m}$ is determined by the value of $v_{j j^{\prime} ; l m}^{l_{2} m_{2} l_{2}^{\prime} m_{2}^{\prime}}(\xi)$ at the grid endpoint, i.e. at $\xi=\xi_{\max }$ and is therefore found to be

$$
\begin{aligned}
\alpha_{j l_{2} m_{2} j^{\prime} l_{2}^{\prime} m_{2}^{\prime}}^{l m}= & \frac{8 \pi}{R} \frac{Q_{l}^{m}\left(\xi_{\max }\right)}{P_{l}^{m}\left(\xi_{\max }\right)}(-1)^{m} \frac{(l-m) !}{(l+m) !} \\
& \times \int P_{l}^{m}(\xi) \rho_{j m_{2} j^{\prime} m_{2}^{\prime}}(\xi) \bar{J}_{l_{2} m_{2} l_{2}^{\prime} m_{2}^{\prime}}^{l m}(\xi) d \xi \\
= & \frac{8 \pi}{R} \frac{Q_{l}^{m}\left(\xi_{\max }\right)}{P_{l}^{m}\left(\xi_{\max }\right)}(-1)^{m} \frac{(l-m) !}{(l+m) !} \delta_{j j^{\prime}} P_{l}^{m}\left(\xi_{j}\right) \\
& \times \bar{J}_{l_{2} m_{2} l_{2}^{\prime} m_{2}^{\prime}}^{l m}\left(\xi_{j}\right)
\end{aligned}
$$


and we can assemble the entire potential as

$$
\begin{aligned}
& V_{j j^{\prime}}^{l_{2} m_{2} l_{2}^{\prime} m_{2}^{\prime}}(\xi, \eta, \phi)= \\
& \sum_{l, m}\left[\sum_{i}-\frac{8 \pi}{R}\left[T_{l m}\right]_{i, j}^{-1} \frac{\delta_{j j^{\prime}}}{\sqrt{w_{j}}} \bar{J}_{l_{2} m_{2} l_{2}^{\prime} m_{2}^{\prime}}\left(\xi_{j}\right) \chi_{i m}(\xi)\right. \\
& \left.+\alpha_{j l_{2} m_{2} j^{\prime} l_{2}^{\prime} m_{2}^{\prime}}^{l m} P_{l}^{m}(\xi)\right] Y_{l}^{m}(\eta, \phi) \text {. } \\
& I=\left(\frac{R}{2}\right)^{6} \int \rho_{i m_{1} i^{\prime} m_{1}^{\prime}}(\xi) Y_{l_{1}}^{m_{1} *}(\eta, \phi) Y_{l_{1}^{\prime}}^{m_{1}^{\prime}}(\eta, \phi) V_{j j^{\prime}}^{l_{2} m_{2} l_{2}^{\prime} m_{2}^{\prime}}(\mathbf{r})\left(\xi^{2}-\eta^{2}\right) d \xi d \eta d \phi \\
& =\frac{\pi R^{5}}{8} \sum_{l=0}^{l_{\max }} \delta_{i i^{\prime}} \delta_{j j^{\prime}} J_{l_{1} m_{1} l_{1}^{\prime} m_{1}^{\prime}}^{l m}\left(\xi_{i}\right)\left[-\frac{\left[T_{l m}\right]_{i, j}^{-1}}{\sqrt{w_{i} w_{j}}}+(-1)^{m} \frac{(l-m) !}{(l+m) !} \frac{Q_{l}^{m}\left(\xi_{\max }\right)}{P_{l}^{m}\left(\xi_{\max }\right)} P_{l}^{m}\left(\xi_{i}\right) P_{l}^{m}\left(\xi_{j}\right)\right] \bar{J}_{l_{2} m_{2} l_{2}^{\prime} m_{2}^{\prime}}\left(\xi_{j}\right),
\end{aligned}
$$

We can now complete the desired expression for the twoelectron integral. Substituting Eq. (37) into Eq. (26) and where $l_{\max }=\min \left(l_{1}+l_{1}^{\prime}+2, l_{2}+l_{2}^{\prime}+2\right)$, the latter identity following from the properties of the $3-j$ symbols that appear in Eqs. (20) and (23).

\section{Double ionization amplitude}

The procedure for constructing the double ionization amplitude follows the development outlined in ref. [9]. The amplitude that gives the triple differential cross section (TDCS) for double photoionization is

$$
\begin{aligned}
& f\left(\mathbf{k}_{1}, \mathbf{k}_{2}\right)=\left\langle\Phi^{(-)}\left(\mathbf{k}_{1}, \mathbf{r}_{1}\right) \Phi^{(-)}\left(\mathbf{k}_{2}, \mathbf{r}_{2}\right)\right| \\
& \quad\left[E+\frac{1}{2} \nabla_{1}^{2}+\frac{1}{2} \nabla_{2}^{2}+\frac{1}{\left|\mathbf{r}_{1}-\frac{\mathbf{R}}{2}\right|}+\frac{1}{\left|\mathbf{r}_{1}+\frac{\mathbf{R}}{2}\right|}\right. \\
& \left.\quad+\frac{1}{\left|\mathbf{r}_{2}-\frac{\mathbf{R}}{2}\right|}+\frac{1}{\left|\mathbf{r}_{2}+\frac{\mathbf{R}}{2}\right|}\right]\left|\Psi_{\mathrm{sc}}^{+}\left(\mathbf{r}_{1}, \mathbf{r}_{2}\right)\right\rangle \\
& \quad \equiv\left\langle\Phi^{(-)}\left(\mathbf{k}_{1}, \mathbf{r}_{1}\right) \Phi^{(-)}\left(\mathbf{k}_{2}, \mathbf{r}_{2}\right)\left|E-H_{0}\right| \Psi_{\mathrm{sc}}^{+}\left(\mathbf{r}_{1}, \mathbf{r}_{2}\right)\right\rangle,
\end{aligned}
$$

where $E=k_{1}^{2} / 2+k_{2}^{2} / 2$ is the total photoelectron energy, $\Psi_{s c}^{+}$is the first-order wave function of Eq. (7) and $\Phi^{(-)}(\mathbf{k}, \mathbf{r})=\Phi^{(+) *}(-\mathbf{k}, \mathbf{r})$ is an $\mathrm{H}_{2}^{+}$continuum eigenfunction with incoming momentum $\mathbf{k}$. The integral in Eq. (39) is performed over the finite, real portion of the underlying grid, i.e. $-1 \leq \eta_{1}, \eta_{2} \leq 1,0 \leq \phi_{1}, \phi_{2} \leq 2 \pi$,
$1 \leq \xi_{1}, \xi_{2} \leq \xi_{0}$, where $\xi_{0}$ lies on the real portion of the ECS grid. Equation (39) has been shown to produce the physical breakup amplitude as $\xi_{0}$ is increased, aside from a knowable, but irrelevant, overall phase [22]. The use of $\mathrm{H}_{2}^{+}$continuum eigenfunctions in Eq. (39) is essential for producing numerically stable results, since their orthogonality to the bound states of $\mathrm{H}_{2}^{+}$eliminates spurious contributions of the single ionization channels that can contaminate the results.

Computation of the $\mathrm{H}_{2}^{+}$continuum eigenfunctions in prolate spheroidal coordinates was detailed in I. $\Phi^{(-)}(\mathbf{k}, \mathbf{r})$ is first partitioned into unperturbed and scattered wave components

$$
\Phi_{\mathbf{k}}^{(+)}(\xi, \eta, \phi)=\Phi_{0}^{(+)}(\xi, \eta, \phi)+\Phi_{s c}(\xi, \eta, \phi),
$$

to get

$$
\left(H-\frac{k^{2}}{2}\right) \Phi_{s c}(\xi, \eta, \phi)=\left(\frac{k^{2}}{2}-H\right) \Phi_{0}^{(+)}(\xi, \eta, \phi) .
$$

We use that fact that the asymptotic form of $\Phi_{0}^{(+)}$is determined by the long-range behavior of the Coulomb interaction and must coincide, for large $\xi$, with the atomic Coulomb function, $\psi_{c}^{(+)}(\mathbf{k}, \mathbf{r})$, with $Z=2$, corresponding to an electron with incoming momentum $\mathbf{k}$. The explicit choice for $\Phi_{0}^{(+)}$is

$$
\Phi_{0}^{(+)}(\xi, \eta, \phi)=\left(\frac{2}{\pi}\right)^{1 / 2} \sum_{l, m} \frac{i^{l} e^{i \eta_{l}}}{k r(\xi, \eta)} g(\xi) \phi_{l, k}^{(c)}(r(\xi, \eta)) Y_{l}^{m}(\hat{\mathbf{r}}(\xi, \eta, \phi)) Y_{l}^{m *}(\hat{\mathbf{k}}),
$$

where $\phi_{l, k}^{(c)}$ is the partial-wave Coulomb function, $\eta_{l}$ is the Coulomb phaseshift, $g(\xi)$ is a cutoff function that goes to zero as $\xi \rightarrow 1$, and the mapping between spherical and 
prolate spheroidal variables is given through

$$
\begin{aligned}
r & =a \sqrt{\xi^{2}+\eta^{2}-1} \\
\cos \theta & =\frac{\xi \eta}{\sqrt{\xi^{2}+\eta^{2}-1}} .
\end{aligned}
$$

The function $g(\xi)$ is introduced in oprder to cut off $\Phi_{0}^{(+)}$ near the one-center singularity at $r=0(\xi=1, \eta=0)$, which is not removed by the use of prolate spheroidal coordinates. With this choice, we can solve the driven equations obtained from Eq. (41) for each partial wave and then construct $\Phi_{\mathbf{k}}^{(+)}(\xi, \eta, \phi)$ any direction of $\mathbf{k}$. Our approach differs from earlier work on $\mathrm{H}_{2}^{+}$continuum states using prolate spheroidal coordinates, as well as the recent work of Serov and Joulakian on $\mathrm{H}_{2}$ DPI, where the asymptotic behavior of the one-electron continuum states was expressed in terms of two-center phaseshifts $[16,19,23-25]$.

The evaluation of Eq. (39) is simplified by taking advantage of the fact that the operator $\left(E-H_{0}\right)$ in that equation gives zero when operating to the left. With $\Psi_{\mathrm{sc}}^{+}\left(\mathbf{r}_{1}, \mathbf{r}_{2}\right)$ expanded as in Eq. (31) along with an analogous expansion of $\Phi^{(-)}\left(\mathbf{k}_{1}, \mathbf{r}_{1}\right) \Phi^{(-)}\left(\mathbf{k}_{2}, \mathbf{r}_{2}\right)$,

$$
\begin{gathered}
\left(\Phi^{(-)}\left(\mathbf{k}_{1}, \mathbf{r}_{1}\right) \Phi^{(-)}\left(\mathbf{k}_{2}, \mathbf{r}_{2}\right)\right)^{*}=\sum_{i j l_{1} l_{2} m} i^{-\left(l_{1}+l_{2}\right)} e^{i \eta_{l_{1}}+i \eta_{l_{2}} \times} \\
Y_{l_{1} m}\left(\hat{\mathbf{k}}_{1}\right) Y_{l_{2} M-m}\left(\hat{\mathbf{k}}_{2}\right) c_{i l_{1} m}^{k_{1}} c_{j l_{2} M-m}^{k_{2}} \Phi_{i l_{1} m}\left(\mathbf{r}_{1}\right) \Phi_{j l_{2} M-m}\left(\mathbf{r}_{\mathbf{2}}\right)
\end{gathered}
$$

the six-dimensional volume integral can thereby be converted into a five-dimensional surface integral, as was done previously in spherical polar coordinates $[1,2]$. The integrals in $\eta_{1}, \phi_{1}, \eta_{2}, \phi_{2}$ can be performed analytically, leaving a surface integral in $\xi_{1}$ and $\xi_{2}$. However in this case, instead of reducing the integral to an integral over an arc of constant $\sqrt{\xi_{1}^{2}+\xi_{2}^{2}}$ by analogy to the polar coordinates formulation, we choose to make the surface a rectilinear one. The surface segments are the lines $1<\xi_{1}<\xi_{0}, \xi_{2}=\xi_{0}$ and $1<\xi_{2}<\xi_{0}, \xi_{1}=\xi_{0}$. Equation (39) can then be evaluated as,

$$
\begin{aligned}
& f\left(\mathbf{k}_{1}, \mathbf{k}_{2}\right)=\left\langle\Phi^{(-)}\left(\mathbf{k}_{1}, \mathbf{r}_{1}\right) \Phi^{(-)}\left(\mathbf{k}_{2}, \mathbf{r}_{2}\right)\left|E-H_{0}\right| \Psi_{\mathrm{sc}}^{+}\left(\mathbf{r}_{1}, \mathbf{r}_{2}\right)\right\rangle= \\
& \sum_{i j l_{1} l_{2} m} \sum_{i^{\prime} j^{\prime} l_{1}^{\prime} l_{2}^{\prime}} c_{i l_{1} m}^{k_{1} m} c_{j l_{2} M-m}^{k_{2}} C_{i^{\prime} j^{\prime} l_{1}^{\prime} l_{2}^{\prime} m} i^{-\left(l_{1}+l_{2}\right)} e^{i \eta_{l_{1}}+i \eta_{l_{2}} Y_{l_{1} m}\left(\hat{\mathbf{k}}_{1}\right) Y_{l_{2} M-m}\left(\hat{\mathbf{k}}_{2}\right)} \\
& \times \delta_{l_{2}, l_{2}^{\prime}} \frac{R^{4}}{32} \int \chi_{i m}(\xi) \chi_{i^{\prime} m}(\xi) S_{m l_{1} l_{1}^{\prime}}(\xi) d \xi\left(\xi_{0}^{2}-1\right)\left[\left.\chi_{j M-m}\left(\xi_{0}\right) \frac{d \chi_{j^{\prime} M-m}}{d \xi}\right|_{\xi=\xi_{0}}-\left.\chi_{j^{\prime} M-m}\left(\xi_{0}\right) \frac{d \chi_{j M-m}}{d \xi}\right|_{\xi=\xi_{0}}\right]+\mathbf{1} \leftrightarrow \mathbf{2},
\end{aligned}
$$

where we have used Eq. (15) in the integration over the angular variables and integration by parts, retaining only the surface terms, for integrals involving derivative terms in $\xi$.

We conclude this section with the working expressions for the DPI cross sections expressed in the velocity gauge. The TDCS for double photoionization of the fixed-nuclei, aligned molecule is given by

$$
\frac{d^{3} \sigma}{d E_{1} d \Omega_{1} d \Omega_{2}}=\frac{4 \pi^{2}}{\omega c} k_{1} k_{2}\left|f\left(\mathbf{k}_{1}, \mathbf{k}_{2}\right)\right|^{2},
$$

while the corresponding single differential (SDCS) or energy sharing cross section is obtained by integrating the TDCS over the ejection angles of the photoelectrons:

$$
\frac{d \sigma}{d E_{1}}=\int d \Omega_{1} d \Omega_{2} \frac{d^{3} \sigma}{d E_{1} d \Omega_{1} d \Omega_{2}} .
$$

The SDCS is easily evaluated as the sum of the squared moduli of the partial-wave amplitudes that determine $f\left(\mathbf{k}_{1}, \mathbf{k}_{2}\right)$. For linearly polarized incident radiation and randomly oriented molecules, the physical SDCS is ob- tained as the weighted average of $\Sigma$ and $\Pi$ contributions:

$$
\frac{d \sigma^{\text {phys }}}{d E_{1}}=\frac{1}{3}\left(\frac{d \sigma^{(\Sigma)}}{d E_{1}}+2 \frac{d \sigma^{(\Pi)}}{d E_{1}}\right) .
$$

The total cross section is the integral of this SDCS from 0 to $E$, the latter being the total photoelectron energy. Note that we differ from some other authors who define the SDCS so that it gives the total cross section when integrated from 0 to $E / 2$.

\section{COMPUTATIONS}

To facilitate comparisons with earlier work, we have carried out fixed-nuclei calculations of $\mathrm{H}_{2}$ DPI at its equilibrium, $R=1.4 \mathrm{bohr}$, for $75 \mathrm{eV}$ photon energy. We have performed calculations at the equilibrium $\mathrm{H}_{2}$ geometry, $R=1.4$ bohr, to compare with our earlier calculations. The $\xi$ grid for these calculations was subdivided into 8 evenly spaced real intervals, each of length 8.0 , running from 1.0 to 65.0 and an additional complex element of length 15.0 and a rotation angle of $30^{\circ}$. We use 14thorder DVR in each interval. For the angular expansion, 


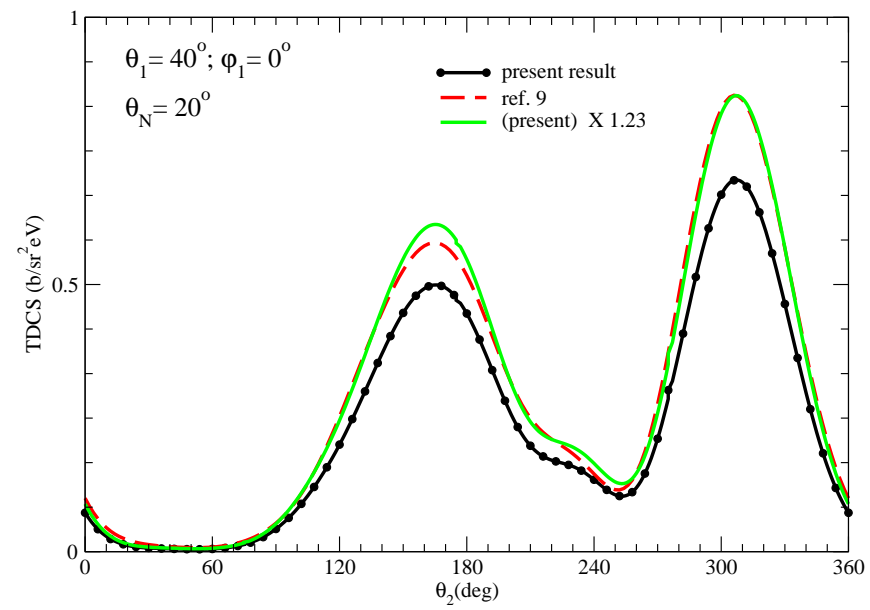

FIG. 1: (Color online) TDCS for double photoionization of $\mathrm{H}_{2}$ for $75 \mathrm{eV}$ photon energy. The molecule, ejected electrons, and polarization vector are coplanar. All angles are measured from the polarization axis. The angle between molecule and polarization axis is $\theta_{\mathrm{mol}}=20^{\circ}$; the angle between fixed electron (with 80 percent of available energy) and polarization axis is $\theta_{1}=40^{\circ}$; the angle between the molecule and the fixed electron is $20^{\circ}$.

we retained terms up to $l_{\max }=6$. The ground-state $\mathrm{H}_{2}$ energy, obtained using only the first two real $\xi$ elements, was -1.17442 hartree, in excellent agreement with the accurate value of -1.17447 hartree results of Wolniewitz [26]. We note that calculations in spherical coordinates reported in ref. [9] using a single-center expansion with $l_{\max }=7$ give a target energy of -1.16908 hartree, which is $0.15 \mathrm{eV}$ higher than the accurate value.

\section{RESULTS}

Figure 1 shows TDCS results for a case previously considered. The two photoelectrons both lie in the plane defined by the molecule and the polarization axis. The angle between the molecule and the polarization axis is $20^{\circ}$, while the fixed electron, with 80 percent of the available energy, is at $40^{\circ}$ to the polarization axis. For this arrangement, the TDCS is small and has roughly equal contributions from the $\Sigma_{u}$ and $\Pi_{u}$ components of the wave function. Evidently, the present results are uniformly smaller than those we reported in ref. [9], which were obtained using a single-center expansion in spherical coordinates. Curiously, a single scaling factor of 1.23 bring the two results into perfect agreement.

It turns out that for any geometry, a simple renormalization of the earlier single-center results brings them into perfect agreement with the present calculations. This is demonstrated with the results displayed in Figs. 2-4, which show results for different orientations of the fixed electron and different energy sharings. The scaling factor needed to bring the present and the earlier calculations into agreement only depends on the energy sharing. That


FIG. 2: (Color online) TDCS for double photoionization of $\mathrm{H}_{2}$ for $75 \mathrm{eV}$ photon energy. The molecule, ejected electrons, and polarization vector are coplanar. The fixed electron, with 20 percent of the available energy, is perpendicular to the polarization axis. $\Theta_{N}$ indicates angle between polarization and molecular axis.
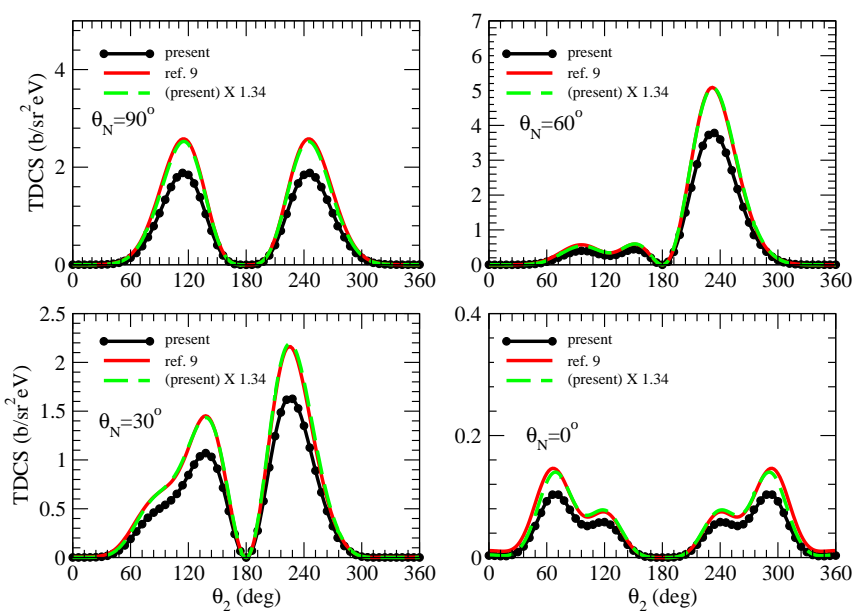

FIG. 3: (Color online) As in Fig. 2, except fixed electron has 50 percent of the available energy.

factor varies from 1.34 for equal energy sharing to 1.23 for 20 percent energy sharing, independent of the relative orientation of photoelectrons and molecular orientation with respect to the polarization axis.

The present results could not be changed by increasing the grid size, the DVR order or the number of angular terms retained in solving for the scattered wave or the $\mathrm{H}_{2}^{+}$continuum functions. A careful convergence study had also been carried out in the earlier single-center ECS study, so the differences seen in the absolute magnitude of TDCS from the two studies was surprising. The observed differences led us to suspect that they might be attributable to differences in the initial-state target wave functions. There is $0.15 \mathrm{eV}$ difference in the groundstate target energies. Since both calculations were car- 

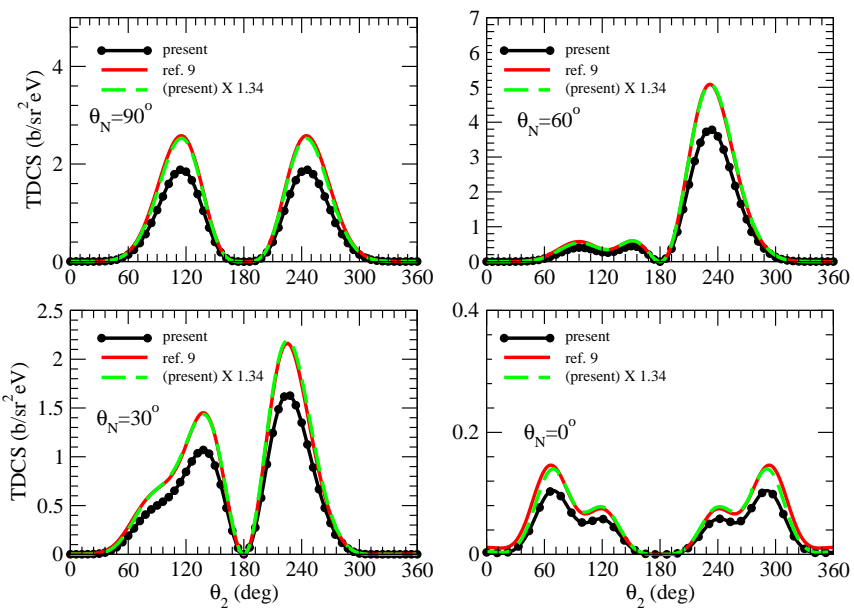

FIG. 4: (Color online) As in Fig. 3, except fixed electron is parallel to polarization axis.

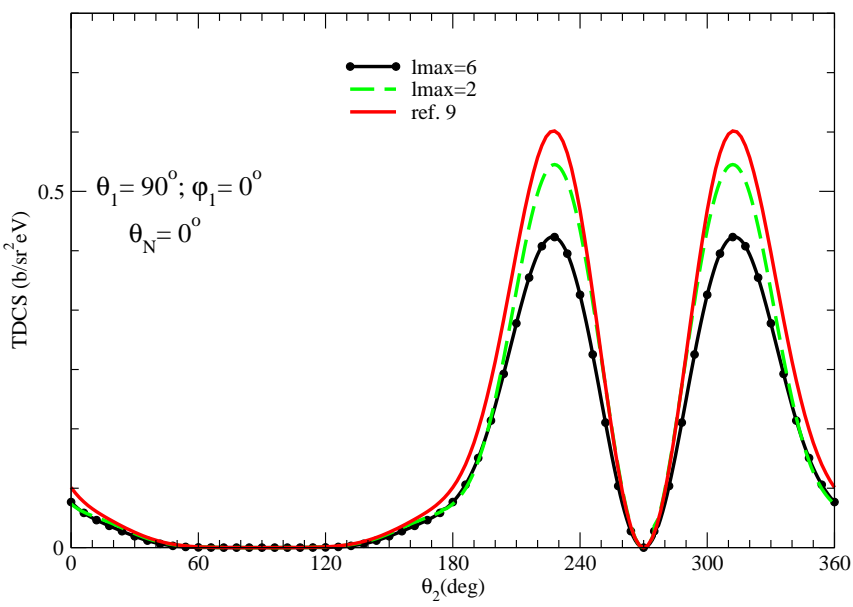

FIG. 5: (Color online) TDCS for $75 \mathrm{eV}$ photon energy and equal energy sharing, with fixed electron perpendicular to molecular axis. Present result is compared with those of ref. 9 and with a calculation in which the initial state is calculated using $l_{\max }=2$ in prolate coordinates.

ried out at $75 \mathrm{eV}$ photon energy, this difference results in a slightly smaller total photoelectron energy in the present calculations. However, we checked that changing the photoelectron energy could not account for the observed differences.

Single-center expansions are notoriously difficult to converge and first-order changes in the wave function only change the energy in second-order. This observation, coupled with the fact that the amplitude for double ionization is a sensitive probe of electron correlation, being effectively zero in an independent particle treatment, led us to suspect that the differences we were observing might be attributable to small differences in the initial state wave functions themselves. To test this hypothesis, we recomputed the initial-state target wave function using $l_{\max }=2$ in the angular expansion, which gave an
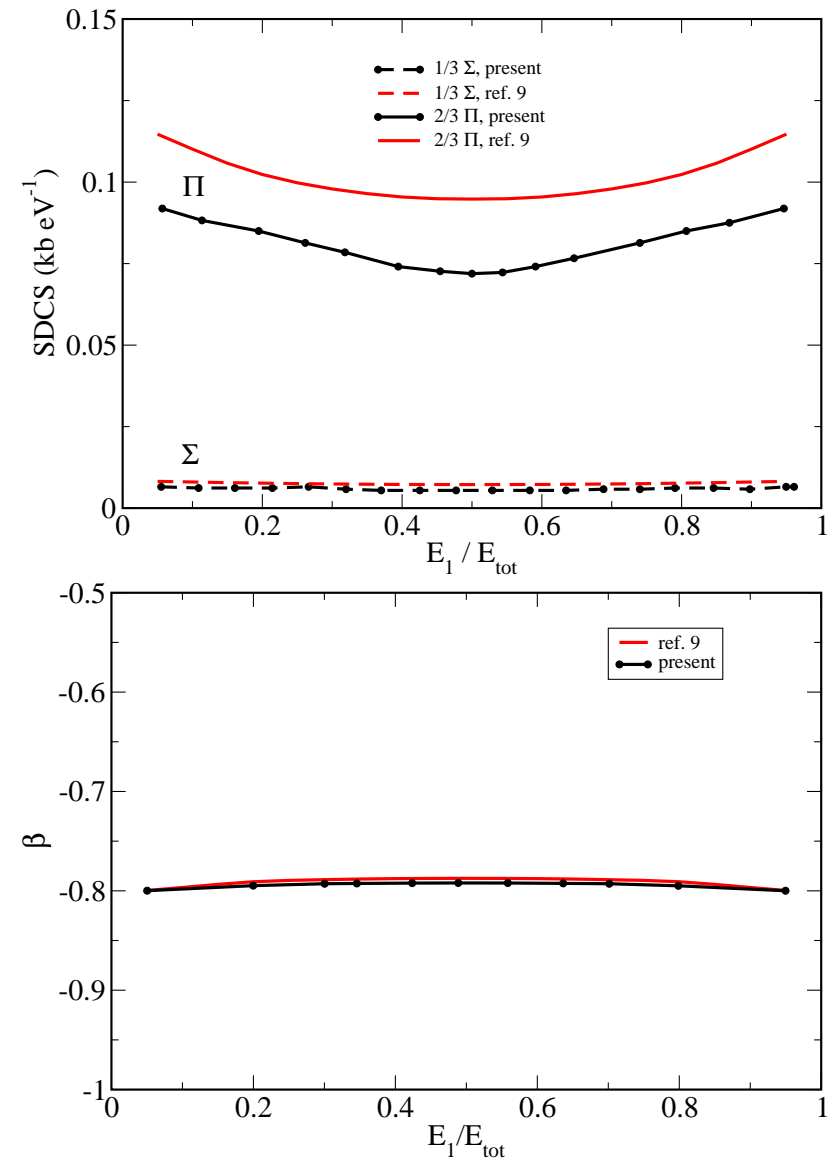

FIG. 6: (Color online) SDCS and $\beta$ parameter for $\mathrm{H}_{2}$ DPI at $75 \mathrm{eV}$ photon energy. Present results are compared with those of ref. 9 .

energy of -1.1732 hartree, only one millihartree higher than the original value. We then solved the driven equation for the scattered wave using this initial-state wave function, but with the original basis set parameters and angular terms up to $l_{\max }=6$ in both $\Psi_{s c}^{+}$and $\Phi^{(-)}$. The results in Fig. 5 show a significant rise in the magnitude of the TDCS resulting from the change in the target state wave function, giving values much closer to those reported in ref. [9]. We conclude that magnitude of the TDCS depends very sensitively on the quality of the initial state wave function, while the relative shape of the angular distribution is less sensitive to small changes in the target-state wave function.

Figure 6 compares the calculated $\Sigma$ and $\Pi$ components of the SDCS, as well as the $\beta$-parameter, with the earlier results. It is easy to verify that the SDCS values differ by precisely the same factors reflected in the TDCS. At equal energy sharing, the ratio is 1.34 , while at $20 / 80$ percent energy sharing, the ratio is 1.23 . The $\beta$-parameter depends only on a ratio of cross sections and thus the present results for this quantity agree well with our earlier values.

The only other theoretical treatments of differential 
DPI cross sections available for comparison are the timedependent close-coupling (TDCC) treatment of Colgan, Pindzola and Robicheaux [10] and the recent PSECS study of Serov and Joulakian [16]. The TDCC study presented TDCS results for the same kinematic arrangements shown in Figs. 2-4. Those results are in excellent agreement with present results in shape, while the magnitudes of the TDCC TDCS are somewhat larger than the present values, but generally smaller than those presented in ref. [9]. Colgan et al. report a value of -0.802 for the $\beta$-parameter at equal energy sharing, which is within $1 \%$ of what we find. However, since they do not report values for the SDCS, we cannot see whether the scaling we have found between integrated cross sections and TDCS applies to their results. The only TDCS values reported by Serov and Joulakian are for the single case shown in Fig. 1. Curiously, their results are in almost perfect agreement, in shape and magnitude, with our earlier single-center ECS results, while their SDCS results are $\sim 8 \%$ larger than the single-center values, or $\sim 38 \%$ larger than the present results. While we can point to an inconsistency between the magnitudes of the TDCS and SDCS reported in the PSECS study, we are at a loss to explain its origin.

\section{DISCUSSION}

The present study extends our development of an exterior scaled, finite-element/ discrete variable representation in prolate spheroidal coordinates to study double ionization of two-electron molecular targets. The present development retains much of the simplicity and efficiency of the FEM/DVR in spherical coordinates. We have shown that an analogous approach for constructing matrix elements of the electronic repulsion potential, $1 /\left|\mathbf{r}_{1}-\mathbf{r}_{2}\right|$, based on solving Poisson's equation in spheroidal coordinates gives a representation which is diagonal in the radial DVR indices and a correspondingly sparse representation of the Hamiltonian. Prolate spheroidal coordinates are a natural choice for studying diatomic targets and the angular expansion of the wave function converges very rapidly in this coordinate system.

We have found that, while the shapes of the fully differential double ionization cross sections converge rapidly and are completely consistent with our earlier calculations, the magnitude of the DPI cross sections shows a surprising sensitivity to small changes in the target wave functions.

To extend the present development to more complex molecular targets, we could adopt an approach similar to that outlined in our two recent studies of double photoionization of $\mathrm{Be}$ and $\mathrm{Li}[27,28]$. The idea would be to use a hybrid basis where all but two active electrons are described using molecular orbitals, while the two active electrons are described using primitive FEM/DVR functions. The molecular orbitals would be expanded in the same underlying DVR basis, but since their radial extent in limited, we could exploit the finite element aspect of the FEM/DVR to use DVR functions that span only the first few elements in constructing the molecular orbitals. The remainder of the DVR grid representation is left untouched and describes the continuum portions of the wave function. Such an approach retains much of the simplicity of the original FEM/DVR approach, while allowing for a straightforward extension of the method to study double ionization of targets with more than two electrons.

\section{Acknowledgments}

This work was performed under the auspices of the US Department of Energy by the University of California Lawrence Berkeley National Laboratory under Contract DE-AC02-05CH11231 and was supported by the U.S. DOE Office of Basic Energy Sciences, Division of Chemical Sciences. CWM acknowledges support from the National Science Foundation (Grant No. PHY-0604628).

This document was prepared as an account of work sponsored by the United States Government. While this document is believed to contain correct information, neither the United States Government nor any agency thereof, nor the Regents of the University of California, nor any of their employees, makes any warranty, express or implied, or assumes any legal responsibility for the accuracy, completeness, or usefulness of any information, apparatus, product, or process disclosed, or represents that its use would not infringe privately owned rights. Reference herein to any specific commercial product, process, or service by its trade name, trademark, manufacturer, or otherwise, does not necessarily constitute or imply its endorsement, recommendation, or favoring by the United States Government or any agency thereof, or the Regents of the University of California. The views and opinions of authors expressed herein do not necessarily state or reflect those of the United States Government or any agency thereof or the Regents of the University of California.
[1] C. W. McCurdy, M. Baertschy, and T. N. Rescigno, J. Phys. B 37, R137 (2004).

[2] D. A. Horner, F. Morales, T. N. Rescigno, F. Martín, and C. W. McCurdy, Phys. Rev. A 76, 030701(R) (2007).
[3] L. Malegat, P. Selles, and A. K. Kazansky, Phys. Rev. A 60, 3667 (1999).

[4] M. S. Pindzola, et al., J. Phys. B 40, R39 (2007).

[5] A. Palacios, T. N. Rescigno, and C. W. McCurdy, Phys. 
Rev. A 77, 032716 (2008).

[6] J. Feist, S. Nagele, R. Pazourek, E. Persson, B. I. Schneider, L. A. Collins, and J. Burgdorfer, Phys. Rev. A. 77, 043420 (2008).

[7] A. Palacios, T. N. Rescigno, and C. W. McCurdy, Phys. Rev. A 79, 033402 (2009).

[8] J. Feist, S. Nagele, R. Pazourek, E. Persson, B. I. Schneider, L. A. Collins, and J. Burgdorfer, Phys. Rev. Lett. 103, 063002 (2009).

[9] W. Vanroose, D. A. Horner, F. Martín, T. N. Rescigno, and C. W. McCurdy, Phys. Rev. A 74, 052702 (2006).

[10] J. Colgan, M. S. Pindzola, and F. Robicheaux, Phys. Rev. Lett. 98, 153001 (2007).

[11] F. Morales, F. Martín, D. A. Horner, T. N. Rescigno, and C. W. McCurdy, J. Phys. B 42, 134013 (2009).

[12] L. Tao, C. W. McCurdy, and T. N. Rescigno, Phys. Rev. A 79, 012719 (2009).

[13] L. Tao, C. W. McCurdy, and T. N. Rescigno, Phys. Rev. A 80, 013402 (2009).

[14] W. Vanroose, F. Martín, T. N. Rescigno, and C. W. McCurdy, Science 310, 1787 (2005).

[15] D. A. Horner, W. Vanroose, T. N. Rescigno, F. Martín, and C. W. McCurdy, Phys. Rev. Lett. 98, 073001 (2007).

[16] V. V. Serov and B. B. Joulakian, Phys. Rev. A 80, 062713
(2009).

[17] T. N. Rescigno and C. W. McCurdy, Phys. Rev. A 62, 032706 (2000).

[18] D. A. Telnov and S.-I. Chu, Phys. Rev. A 76, 043412 (2007).

[19] L. I. Ponomarev and L. N. Somov, J. Comp. Phys. 20, 183 (1976)

[20] A. C. Wahl, P. E. Cade, and C. C. J. Roothaan, J. Chem. Phys. 41, 2578 (1964).

[21] M. Abramowitz and I. A. S. C. Slater, Handbook of Mathmatical Functions (Dover, New York, 1965).

[22] T. N. Rescigno, M. Baertschy, and C. W. McCurdy, Phys. Rev. A 68, 020701(R) (2003).

[23] D. I. Abramov, A. Y. Kazakov, L. I. Ponomarev, S. Y. Slavyanov, and L. N. Somov, J. Phys. B 12, 1761 (1979).

[24] J. Rankin and W. R. Thorson, J. Comp. Phys. 32, 437 (1979).

[25] Y. S. Tergiman, Phys. Rev. A 48, 88 (1993).

[26] L. Wolniewicz, J. Chem. Phys. 103, 1792 (1995).

[27] F. Yip, C. W. McCurdy, and T. N. Rescigno, Phys. Rev. A 81, 053407 (2010).

[28] F. Yip, C. W. McCurdy, and T. N. Rescigno, Phys. Rev. A 82, 063419 (2010). 